\title{
TAGUNG
}

\section{Zum Verhältnis von Demokratie und europäischer Integration}

\author{
Katharina Joho*
}

Die Begrifflichkeiten der Demokratie und der europäischen Integration werden in der Wissenschaft kontrovers diskutiert. Was bedeutet eigentlich Demokratie und was versteht man unter der europäischen Integration und letztlich ihrer Finalität? Der Umgang mit diesen Begrifflichkeiten und ihrem Verhältnis zueinander hängt stark davon ab, an welchem Leitbild man sich orientiert. Entspricht dieses mehr dem Ideal des demokratischen Nationalstaates des 19. Jahrhunderts oder aber einem der vielfältigen postmodernen Konzepte wie zum Beispiel dem der Multi-Level-Governance-Theorie. Auch die Disziplin des forschenden Wissenschaftlers spielt eine nicht unerhebliche Rolle. Nicht zuletzt deswegen ist die Debatte um die Verbindung von europäischer Integration und Demokratie und die oft geäußerte Prognose eines europäischen Demokratiedefizits so vielfältig und schwierig.

Demokratie und Europäische Union so lautete der schlichte Titel der IX. gemeinsamen interdisziplinären Tagung der Schweizerischen Studiengesellschaft für die Europäische Integration (ECSA Suisse), der Österreichischen Gesellschaft für Europaforschung (ECSAAustria) und des Arbeitskreises Europäische Integration e.V. Deutschland (AEI e.V.). In verschiedenen Beiträgen äußerten sich Politikwissenschaftler, Juristen und Ökonomen aus verschiedenen europäischen Ländern zum Zustand der Demokratie in der Europäischen Union, möglichen Reformen und ihren Rückwirkungen auf die nationalen Systeme der EU-Mitgliedstaaten sowie auf mit der Euro-

\section{Die EU und Demokratie}

Dreiländertagung der Österreichischen Gesellschaft für Europaforschung (ECSA-Austria), des Arbeitskreises Europäische Integration e.V. Deutschland (AEI) und der Schweizerischen Studiengesellschaft für die Europäische Integration (ECSA Suisse), an der Universität Luzern

Luzern, 17./18. Oktober 2008

\section{Begrüßung}

Prof. Dr. Sandra LAVENEX, Vorsitzende ECSA Suisse; Universität Luzern

\section{Demokratie in der EU I}

Vorsitz: Prof. Dr. René SCHWOK, Universität Genf

Der Wandel der Vorstellung über das Verhältnis von Integration und Demokratie seit dem Beginn der Integration

Prof. Dr. Heinrich SCHNEIDER, Universität Wien

Der Reformvertrag und die Demokratie

Prof. Dr. Stefan GRILLER, Europainstitut an der Wirtschaftsuniversität Wien

Institutionelle Ansätze zur Lösung des Demokratiedefizits

Dr. Stefanie BAILER, Universität Zürich

Demokratie in der EU II

Vorsitz: Prof. Dr. Dr. h.c. mult. Peter-Christian MÜLLER-GRAFF, Universität Heidelberg

Schaffung einer europäischen Öffentlichkeit Prof. Dr. Hans-Jörg TRENZ, ARENA Centre for European Studies, Universität Oslo

Interaktion von EU und Demokratie im Rahmen der Theorie über Multilayered Governance Prof. Dr. Thomas COTTIER, Universität Bern

* Katharina Joho, Geschäftsführerin des Arbeitskreises Europäische Integration e.V.; Wissenschaftliche Mitarbeiterin, Otto-Suhr-Institut für Politikwissenschaft, Freie Universität Berlin. 
päischen Union in Beziehung stehende Drittstaaten.

\section{Zum Verhältnis von Demokratie und europä- ischer Integration}

Zunächst skizzierte Heinrich Schneider den allgemeinen Wandel der Vorstellungen von Demokratie im Verlauf der europäischen Integration. Während sich die europäischen Föderalisten zu Beginn der europäischen Einigung nach dem Zweiten Weltkrieg am Ideal des föderalen Bundesstaates und damit der etatistischen Version von Demokratie verorteten, bestimmte nach dem Scheitern der Europäischen Politischen Gemeinschaft und der Europäischen Verteidigungsgemeinschaft in den 1950er Jahren der Siegeszug der ,Methode Monnet' die Sicht auf das europäische Konstrukt. Überlegungen zur Demokratiefähigkeit der Europäischen Wirtschaftsgemeinschaft und vor allem zu ihrer Legitimität rückten in den Hintergrund und das Europäische Parlament fristete ein Schattendasein.

Erst mit der Aufgabenerweiterung der Europäischen Wirtschaftsgemeinschaft in den 1960er Jahren sei es zu einem Ruf nach mehr demokratischer Legitimation gekommen und das Demokratiethema damit zum ,evergreen geworden. Nach Auffassung von Schneider orientieren sich auch die heutigen Vorschläge noch im Wesentlichen an den Reformideen und Leitvorstellungen der 1960er Jahre.

Wie sich die Demokratie in der Europäischen Union aus der Sicht des relativ jungen sogenannten Multi-Layered-Governance-Ansatzes darstellt, zeigte Thomas Cottier aus rechtswissenschaftlicher Perspektive in seinem Vortrag auf.

Entgegen der strengen traditionellen Trennung zwischen nationalstaatlicher Souveränität und anderen Räumen des Rechts müsse man sich das Verhältnis der verschiedenen Rechtsebenen als fünfstöckiges Haus vorstellen, bestehend aus kommunalen Verfassungen, Länderverfassungen, nationalen Verfassungen, europäischem Gemeinschaftsrecht
Auswirkungen der Europäisierung auf die Demokratie in Mitglied- und Nichtmitgliedstaaten

Vorsitz: Prof. Dr. Fritz BREUSS, Europainstitut an der Wirtschaftsuniversität Wien

Mehr Demokratie durch Frühwarnung?

Daniela KIETZ, Stiftung Wissenschaft und Politik, Berlin

Lessons the EU could draw from the Swiss case in order to develop its own public sphere

through legislative referendums

Prof. Dr. René SCHWOK, Universität Genf

Is there a European impact on the domestic policy process of small states?

Prof. Dr. Ioannis PAPADOPOULOS, Alexandre AFONSO, Marie-Christine FONTANA, Universität Lausanne

Externe Demokratieförderung der EU

Vorsitz: Prof. Dr. Joachim BLATTER, Universität Luzern

Externe Demokratieförderung der EU in der sektoralen Perspektive

Prof. Dr. Sandra LAVENEX, Universität Luzern; Prof. Dr. Frank SCHIMMELFENNIG, ETH Zürich; Tina FREYBURG, ETH Zürich

Demokratieförderung im Mittelmeerraum Vera van HÜLLEN, Freie Universität Berlin

\section{Schlussfazit}

und Völkerrecht. All diese Rechtsebenen hätten die Einheit normativer Zielsetzungen gemeinsam, vergleichbare Grundfunktionen und würden einen Vertrauensschutz gewährleisten. Hinsichtlich der Frage nach der Legitimität des Rechts geht Cottier davon aus, dass sich dieses durch verschiedene Komponenten legitimiere. Die demokratische Legitimation stelle zwar das zentrale Standbein dar, sei aber nicht das einzige. Weitere Komponenten wie die Friedensfunktion, die Menschenrechte und allgemeinen Rechtsgrundsätze sowie die Rationalität des Rechts würden miteinander interagieren. Es sei damit von einer Pluralität der Legitimationsquellen auszugehen, welche auf den internationalen, europäischen und nationalen Rechtsebenen in jeweils unterschied- 
licher Ausprägung - mal mehr mal weniger zur Geltung kämen und in einem System der ,checks and balances' miteinander reagierten. Letzteres sei besonders wichtig, da jede Ebene versagen könne, durch das Verhältnis der Gemeinwesen zueinander aber ein Rechtsschutz gewährt werde. Cottier nennt hier das Beispiel der Solange-Rechtsprechung ${ }^{1}$ des deutschen Bundesverfassungsgerichts, welches sich in der Grundrechtsprechung einen Souveränitätsvorbehalt geschaffen habe, und damit einen absoluten Vorrang europäischen Gemeinschaftsrechts verhindere. Cottier schloss mit der Frage, ob hinsichtlich seiner Thesen dann eine Ausweitung der Mehrheitsentscheidungen auf der europäischen Ebene - wie häufig vorgeschlagen - überhaupt noch die Antwort auf das ,Demokratieproblem' sein könne.

Reformansätze für mehr Demokratie im Vertrag von Lissabon: Europäischer oder nationaler Parlamentarismus?

Inwiefern der Vertrag von Lissabon eine Antwort auf das Demokratieproblem darstellen könnte erläuterten aus rechtswissenschaftlicher Perspektive Stefan Griller und aus politikwissenschaftlicher Sicht Daniela Kietz und beleuchteten die Rolle des Europäischen Parlaments und der nationalen Parlamente.

So stellte zunächst Griller die Frage, ob sollte der Vertrag von Lissabon in Kraft treten - er das vielerorts kritisierte Demokratiedefizit lösen könne. Er betonte dabei die große Bedeutung der Stärkung des Europäischen Parlaments für die demokratische Legitimation europäischer Rechtsakte und zeigte drei Reformelemente des Vertrags von Lissabon auf, die dem Legitimationsanliegen Rechnung trügen. Er wies erstens auf die in Titel II des Vertrags über die Europäische Union ${ }^{2}$ genannten demokratischen Grundsätze und das
Instrument der europäischen Bürgerinitiative hin. Zweitens, konstatierte er, seien im Vertrag von Lissabon hinsichtlich der Stärkung des Europäischen Parlaments hin zu einem dem Rat gleichberechtigten Partner bedeutsame Schritte verwirklicht worden. Ferner nannte er das dem Vertrag angehängte sogenannte Subsidiaritätsprotokoll, ${ }^{3}$ das der Stärkung der nationalen Parlamente dienen solle. Vor allem die ersten beiden Elemente stellen für Griller einen bemerkenswerten, wenn auch nicht revolutionären Fortschritt in Richtung einer besseren Legitimierung europäischer Rechtsakte dar.

Mit dem dritten Element, dem Subsidiaritätsprotokoll für die nationalen Parlamente, und damit der Einführung eines sogenannten Subsidiaritäts-Frühwarnsystems, befasste sich Kietz in ihrem Vortrag und analysierte, ob dieser Mechanismus dem System der Europäischen Union wirklich mehr Demokratie bringe. Nach allgemeiner Auffassung gebe es zwei Wege, die Mitwirkung der nationalen Parlamente an der europäischen Gesetzgebung auszuweiten. Erstens könnten die nationalen Parlamente auf nationaler Ebene gestärkt werden und damit indirekt über ihre nationalen Regierungen mehr an der europäischen Gesetzgebung beteiligt werden. Zweitens könnte ihre Beteiligung über die Verankerung neuer Mitwirkungsrechte in den europäischen Verträgen ausgeweitet werden. Während nach Auffassung von Kietz ein Konsens darüber herrsche, dass Ersteres Sache der Nationalstaaten sei, gäbe es keine einheitliche Meinung darüber, ob eine direkte Beteiligung der nationalen Parlamente überhaupt erwünscht sei und, wenn ja, in welcher Weise. So berge insbesondere das sogenannte Subsidiaritäts-Frühwarnsystem einige Probleme. Dieses verfolge ein seinem Zweck der Überprüfung der Subsidiarität der europäischen Gesetzesvorhaben - gegensätzliches

1 Zum Solange I-Urteil siehe BVerfGE 37, 271 und zum Solange II-Urteil siehe BVerfGE 73, 339.

2 Vertrag über die Europäische Union in der konsolidierten Fassung des Lissabonner Vertrages, in: Amtsblatt der EU, Nr. C 115 vom 9. Mai 2008.

3 Protokoll (Nr. 2) über die Anwendung der Grundsätze der Subsidiarität und der Verhältnismäßigkeit, in: Amtsblatt der EU, Nr. C 115 vom 9. Mai 2008, S. 206-209. 
Ziel, nämlich die Stärkung der nationalen Parlamente. So könnte bei der Anwendung des Protokolls die Überprüfung der Subsidiarität leicht zur Nebensache, die Überprüfung des Inhalts der Gesetzesvorhaben zur Hauptsache werden und damit zum möglichen Instrument der nationalen Parlamente, vor allem aber auch ihrer nationalen Regierungen und der Opposition, um ungewollte europäische Gesetzgebungsvorhaben zu hemmen.

Neben der Gefahr der Instrumentalisierung der nationalen Parlamente über das Subsidiaritätsprotokoll sei aber auch auf die verschiedenen, den nationalen Parlamenten zur Verfügung stehenden Ressourcen zu verweisen. So könnten die als stark zu qualifizierenden Parlamente das Subsidiaritätsprotokoll zwar zur Ausweitung ihrer Mitspracherechte nutzen, seien aber eigentlich nicht darauf angewiesen, während die schwachen Parlamente es zwar benötigten, aber aufgrund ihrer beschränkten Ressourcen kaum nutzen könnten. Damit - so Kietz - überwögen die negativen Folgen, wie die der Gefahr der Instrumentalisierung und des negativen Diskurses über die europäische Gesetzgebung, die positiven Folgen. Das Frühwarnsystem sei für das Ziel der Ausweitung der demokratischen Beteiligung der nationalen Parlamente als nicht effizient zu qualifizieren. Der bessere Weg führe über die indirekte Stärkung der nationalen Parlamente innerhalb der nationalen Systeme und die ohnehin nicht aufzuhaltende und in weiten Teilen schon verwirklichte Vernetzung der nationalen Parlamente in der Konferenz der Ausschüsse für Gemeinschafts- und Europaangelegenheiten der nationalen Parlamente in der Europäischen Union (COSAC).

Auch die Politikwissenschaftlerin Stefanie Bailer befasste sich mit dem europäischen Parlamentarismus und fragte, ob die Osterweiterung zu einer Verlangsamung der europäischen Entscheidungsprozesse oder gar Verschiebung der Machtverhältnisse innerhalb des Europäischen Parlaments geführt habe. Sie skizzierte drei mögliche Szenarien: erstens die Oligarchisierung des Europäischen
Parlaments, zweitens die Formalisierung der Entscheidungsprozesse und drittens die Adaptation der neuen Parlamentarier an die schon im Europäischen Parlament praktizierten Entscheidungsprozesse. Weder für die Oligarchisierung noch für die Formalisierung gäbe es bisher viel Evidenz, denn das Abstimmungsverhalten der EU-Parlamentarier habe sich nicht wesentlich geändert und auch die Fraktionsdisziplin scheine überraschenderweise nicht beeinträchtigt. Als Erklärung hierfür nannte sie das letzte Szenario, die Adaptation, gepaart mit einer gesteigerten Integrationsleistung der Fraktionsvorstände. Diese beiden Faktoren seien auch schon bei vorhergehenden Erweiterungen zu beobachten gewesen.

\section{Von der europäischen Öffentlichkeit zur Schaffung direktdemokratischer Elemente}

Mit dem Zusammenhang von europäischer Öffentlichkeit und Demokratie befassten sich ebenfalls aus politikwissenschaftlicher Perspektive Hans-Jörg Trenz und René Schwok.

Einig waren sich beide Wissenschaftler in der Feststellung, dass von einer europäischen Öffentlichkeit oder gar einem europäischen Demos noch lange nicht gesprochen werden könne. Sie zeigten verschiedene Strategien zur Schaffung einer europäischen Öffentlichkeit und ihre Auswirkungen auf.

So äußerte sich zunächst Trenz kritisch über den Kausalzusammenhang zwischen der Schaffung von Öffentlichkeit und Demokratie, der durchaus zweischneidig sei, und fragte, inwiefern das Fehlen von intermediären Strukturen überhaupt ein Hindernis für die Demokratie in der Europäischen Union sei und ob die Schaffung einer europäischen Öffentlichkeit möglich sei. Deren Fehlen sei in der Vergangenheit vor allem von den europäischen Eliten als Problem prognostiziert worden, eine Mobilisierung von unten, also ausgehend vom Volk fehle aber noch weitestgehend. Insbesondere die Möglichkeit der Schaffung einer europäischen Öffentlichkeit über den Prozess einer europäischen Verfas- 
sungsgebung wird von Trenz äußerst kritisch gesehen. So hätten sich die Eliten mittlerweile mehr und mehr in ihr eigenes Demokratieversprechen verstrickt und damit unrealistische Reformerwartungen bei den europäischen Bürgern geweckt. Diese hätten wiederum zu negativen Diskursen über die Europäische Union und ihre weitere Konstitutionalisierung geführt. Eine europäische Öffentlichkeit sei demnach keine Konsensmaschine und führe möglicherweise zu anderen Ergebnissen, als von den Eliten erwartet. Sie könne gar zu einer Ablehnung der europäischen Integration führen.

Schwok sprach sich im Gegensatz zu Trenz eindeutig für die Schaffung einer europäischen Öffentlichkeit aus. Seiner Ansicht nach kann die Einführung direktdemokratischer Elemente im politischen Entscheidungssystem der Europäischen Union zur Schaffung eines europäischen Raumes für die verschiedenen Völker Europas, damit zu einem besseren Identitätsgefühl und schlussendlich $\mathrm{zu}$ mehr Legitimität beitragen. Dies habe das Beispiel der Schweiz gezeigt. Konkret forderte Schwok die Einführung eines optionalen legislativen Referendums auf EU-Ebene mit einfacher Mehrheit ohne Quorum. Dieses könne sich allerdings nur auf diejenige europäische Gesetzgebung beziehen, die bereits vom Rat und vom Europäischen Parlament beschlossen worden sei und müsse einen rechtsverbindlichen Charakter haben. Dieses Referendum mit seiner einfachen Mehrheit könnte dabei helfen, das Interesse der Menschen an der europäischen Integration zu wecken, ihre Partizipation am Entscheidungsprozess zu motivieren, und damit zur Schaffung einer europäischen Öffentlichkeit beitragen.

\section{Wechselwirkungen europäischer Integration mit nationalen Regierungssystemen}

Was das europäische System und seine Implikationen für die nationalen demokratischen Systeme bedeutet und inwiefern die Europäische Union als ,Demokratisator' in Drittländern wirken kann, damit befassten sich Ioan- nis Papadopoulos, Marie-Christine Fontana und Alexandre Afonso; Sandra Lavenex, Frank Schimmelfennig und Tina Freyburg sowie Vera van Hüllen.

Zunächst stellten Papadopoulos, Fontana und Afonso ihr Forschungsprojekt über den Einfluss der europäischen Integration auf die nationalen politischen Entscheidungsprozesse kleiner Staaten vor. Konkreter Untersuchungsgegenstand war hier die Auswirkung der Europäisierung auf die Beziehungen zwischen Exekutive und Legislative und die Entscheidungsfindung im Hinblick auf die Beteiligung korporatistischer Strukturen in der Schweiz, Österreich, Belgien und Irland anhand von fünf Politikbereichen. Betrachtet wurden die Politikbereiche Pensionen, Arbeitslosigkeit, Freizügigkeit, Wettbewerb und Liberalisierung des Elektrizitätssektors.

Vor allem hinsichtlich der Schweiz präsentierten sie schon erste Ergebnisse. Obwohl deren Nicht-Mitgliedschaft die Gefahr eines notorischen Demokratieverlustes im Fall von autonomem Nachvollzug des EU-Rechts berge, zeige ihre vergleichende Studie, dass die Europäisierung nicht generell zu einem Erstarken der Exekutive vis-à-vis der Legislative und den zivilgesellschaftlichen Akteuren führe, sondern dass letztere unter manchen Umständen sogar gestärkt aus dem Europäisierungsprozess hervorgingen.

Bezüglich der Rolle des europäischen Integrationsprozesses bei der Konsolidierung neuer Demokratien zeigte Freyburg anschließend unterstützt von Lavenex und Schimmelfennig - in ihrem Vortrag zwei verschiedene Mechanismen auf, durch die die Europäische Union über den Kreis der Beitrittskandidaten hinaus Demokratie fördere. Dies sei erstens der sogenannte Leverage-Ansatz, wie er auch hinsichtlich der Erweiterungspolitik zum Tragen komme, und zweitens der Linkage-Ansatz im Sinne der Förderung transnationaler Austauschprozesse mit den jeweiligen Zivilgesellschaften. Lavenex, Schimmelfennig und Freyburg untersuchten den Einfluss dieser 
Mechanismen und ihrer Vor- und Nachteile anhand von drei Länderbeispielen, nämlich Marokko, Moldau und der Ukraine.

Van Hüllen beschäftigte sich abschließend mit der Thematik der Demokratieförderung im Mittelmeerraum und setzte sich mit der Frage auseinander, wie autoritäre Regime auf die Demokratisierungsansätze der Europäischen Union reagieren und welche Anreize sie überhaupt dazu bringen, sich darauf einzulassen. Aus der Präsentation der ersten Ergebnisse ging hervor, dass die untersuchten Staaten unterschiedlich auf die EU-Demokratisierungspolitik reagierten und dies auf das Zusammenspiel dreier Faktoren zurückzuführen sei, nämlich Kosten-Nutzen-Kalküle, Adaptationsfähigkeit und die Passfähigkeit zwischen den nationalstaatlichen und den europäischen Normen.

\section{Schlussbemerkung}

Das Verhältnis von Demokratie und europäischer Integration wurde auf der Tagung aus verschiedenen Blickwinkeln heraus erläutert. Es ist deutlich geworden, wie vielfältig die
Debatte hierüber ist und wie kontrovers sie vor allem im interdisziplinären Rahmen bleibt.

Zwar war der Großteil der beteiligten Wissenschaftler und Wissenschaftlerinnen sich dahingehend einig, dass die Europäische Union hinsichtlich ihrer Demokratiefähigkeit noch fehlerhaft sei. Schon hinsichtlich der ersten Lösungsansätze ergaben sich aber große Differenzen, die - so die allgemeine Auffassung - vor allem in den Begrifflichkeiten und den damit verbundenen Herangehensweisen der verschiedenen Disziplinen zu suchen sind.

So bleibt zu konstatieren, dass ohne die interdisziplinäre Definition von Schlüsselbegriffen einer fachübergreifenden Europawissenschaft ein kohärenter Umgang mit den europäischen Problemen nur schwer zu bewerkstelligen ist. Bevor nicht das Verständnis von Demokratie und europäischer Integration sowie ihr Verhältnis zueinander einer interdisziplinären Klärung unterworfen werden, wird das europäische Demokratieproblem wohl ungelöst bleiben.

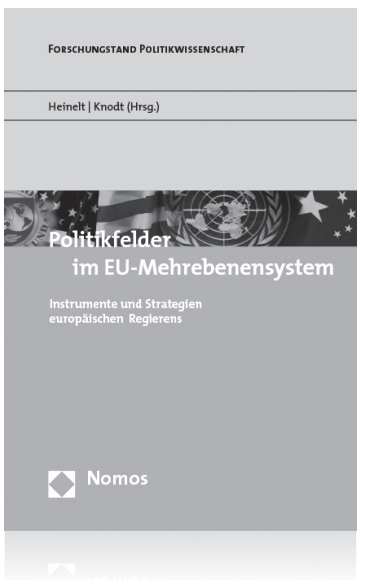

Bitte bestellen Sie im Buchhandel oder versandkostenfrei unter $\boldsymbol{w}$ ww.nomos-shop.de

\section{Politikfelder im EU-Mehrebenensystem} Instrumente und Strategien europäischen Regierens Herausgegeben von Prof. Dr. Hubert Heinelt und Prof. Dr. Michèle Knodt

2008, 334 S., brosch., 29,- €, ISBN 978-3-8329-2273-3 (Forschungsstand Politikwissenschaft)

Dieser Sammelband gibt einen Überblick über die zentralen Politikfelder der EU. Dabei wird jeweils auf die wesentlichen Steuerungsmechanismen und die maßgeblichen Akteure bei der Politikformulierung und Implementation eingegangen und nach der konkreten Ausprägung und Bedeutung einer Mehrebenenstruktur gefragt.

Nomos 